\title{
Subcritical Equilibria in Taylor-Couette Flow
}

\author{
Kengo Deguchi, ${ }^{1,2, *}$ Alvaro Meseguer, ${ }^{3}$ and Fernando Mellibovsky ${ }^{4, *}$ \\ ${ }^{1}$ Department of Mathematics, Imperial College London, South Kensington Campus, London SW7 2AZ, United Kingdom \\ ${ }^{2}$ Department of Aeronautics and Astronautics, Graduate School of Engineering, Kyoto University, Kyoto 606-8501, Japan \\ ${ }^{3}$ Departament de Física Aplicada, Universitat Politècnica de Catalunya, 08034 Barcelona, Spain \\ ${ }^{4}$ Castelldefels School of Telecom and Aerospace Engineering, Universitat Politècnica de Catalunya, 08034 Barcelona, Spain
}

(Received 8 October 2013; published 8 May 2014)

\begin{abstract}
Nonlinear equilibrium states characterized by strongly localized vortex pairs are calculated in the linearly stable parameter region of counterrotating Taylor-Couette flow. These subcritical states are rotating waves whose region of existence is consistent with the critical threshold for relaminarization observed in experiments. For sufficiently rapid outer cylinder rotation the solutions extend beyond the static inner cylinder case to corotation, thus exceeding, for the first time, the boundary defined by the inviscid Rayleigh's stability criterion.
\end{abstract}

DOI: 10.1103/PhysRevLett.112.184502

PACS numbers: 47.20.Qr, 47.20.Ft, 47.27.Cn, 47.32.-y

Rotating fluid motion occurs in extremely diverse situations, ranging from swirl mixing in microfluidic junctions to astronomical accretion disks. For nearly a century, Taylor-Couette flow (TCF), the flow between differentially rotating coaxial cylinders, has provided a canonical prototype to analyze shear and centrifugal fluid instabilities. This has led to countless number of studies for varying values of geometrical parameters such as the aspect ratio $\Gamma=$ $H /\left(r_{o}-r_{i}\right)$ and the radius ratio $\eta=r_{i} / r_{o}$, with $r_{i}$ and $r_{o}$ the inner and outer cylinder radii and $H$ the apparatus height [Fig. 1(a), see also [1], and references therein].

Rayleigh's stability criterion [2] establishes that rotating shear flows are stable whenever the modulus of the angular momentum increases outwards. This criterion considers centrifugal inviscid instability to axisymmetric disturbances and is widely accepted as the origin of Taylor vortex flow in TCF. By using the conventional definition of inner and outer cylinder Reynolds numbers $R_{i}=\left(r_{o}-r_{i}\right) r_{i} \Omega_{i} / \nu$ and $R_{o}=\left(r_{o}-r_{i}\right) r_{o} \Omega_{o} / \nu$, with $\Omega_{i}$ and $\Omega_{o}$ the angular velocities of the cylinders and $\nu$ the kinematic viscosity of the fluid, the laminar base state, known as circular Couette flow (CCF), is Rayleigh stable whenever $\Omega_{o} / \Omega_{i}>$ $\left(r_{i} / r_{o}\right)^{2}$, i.e., $R_{i}\left(R_{o}-\eta R_{i}\right)>0$ [3]. Thus, inviscid stability is to be expected in the $\left(R_{o}, R_{i}\right)$-parameter space region delimited between the straight line $R_{o}=\eta R_{i}$ and the $R_{i}=0$ axis (shaded region in Fig. 2). In practice, CCF remains linearly stable for some extended region beyond the theoretical inviscid stability boundaries due to viscous stabilization. Above the viscous linear stability boundary (dashed curve in Fig. 2), infinitesimally small disturbances grow and saturate, eventually leading to rich patternformation phenomena.

Experiments carried out nearly three and five decades ago reported turbulent motion in the rapidly counterrotating case under conditions for which the flow was expected to be laminar due to the absence of linear instability [4]. Their results, summarized in Fig. 2 by relaminarization boundaries (blue triangles), delimit a hysteretical region where laminar and turbulent flow can both occur and transition is the result of finite-amplitude perturbations. These subcritical turbulent states seem to extend into the Rayleigh-stable region beyond $R_{i}=0$, where the centrifugal instability mechanism is absent. The study of nonlinear instability below the linear stability boundary and further within the Rayleigh-stable region is of paramount importance to the identification of transition mechanisms other than centrifugal in rotating fluids. Astrophysical accretion disks constitute a relevant example of flow instability in the Rayleigh stable regime of rotation [5].

Despite decades of research, turbulence sustainment in subcritical TCF remains unexplained [6]. The common hindrance encountered in lab experiments concerns the unavoidable end cap effects of feasible apparatuses [7]. Recent numerical studies with imposed axial periodicity have reported relaminarization boundaries consistent with those observed in the experiments for moderate values of $R_{i}$ [8]. However, there is no corresponding study extending to the static inner cylinder case.

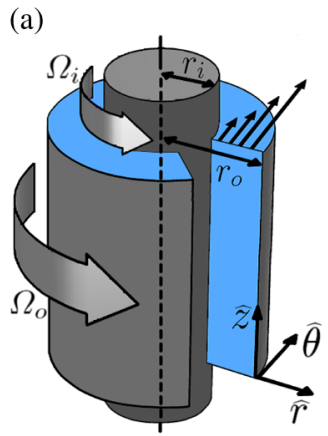

(b)

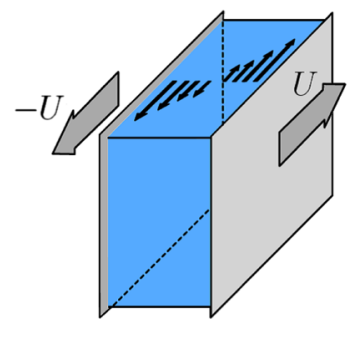

FIG. 1 (color online). Sketch of (a) Taylor-Couette flow and (b) plane Couette flow. 


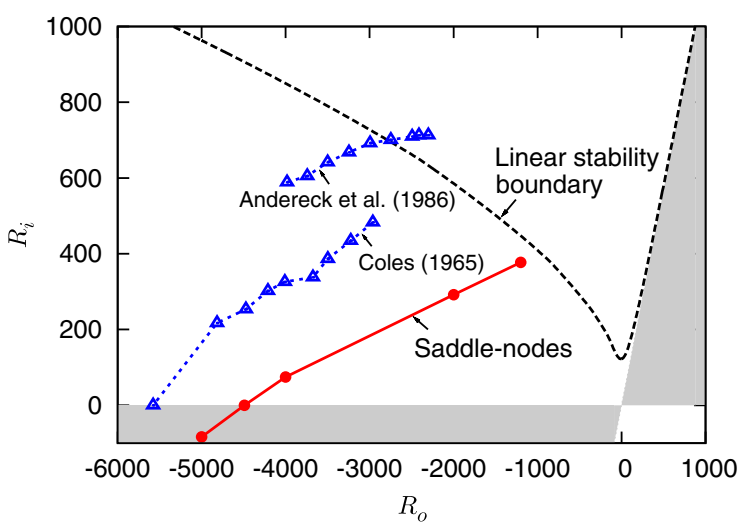

FIG. 2 (color online). Stability diagram of TCF for $\eta=0.883$. Note that $\left(R_{i}, R_{o}\right)$ and $\left(-R_{i},-R_{o}\right)$ correspond simply to turn upside down the TCF setup. The shaded region is Rayleigh stable. The base laminar flow, CCF, is linearly stable below the dashed curve representing the viscous linear stability boundary, while turbulence may be sustained above the line delimited by blue triangles [4]. The red filled circles indicate the saddle-nodes loci corresponding to the nonlinear solutions presented in this Letter.

In this Letter, we attempt to address a theoretical description of subcritical instabilities in TCF by means of dynamical systems theory applied to highly accurate spectral discretizations of the Navier-Stokes equations. The major complication in determining subcritical transition thresholds is that they depend on the size and shape of the disturbances triggering the instability. The approach taken here consists in directly searching for nonlinear solutions as potential precursors to the turbulent state. The interrelations among such states and increasingly complex states bifurcated from them set the stage for the formation of a chaotic set capable of sustaining turbulent motion [9].

The simplest nonlinear Navier-Stokes solutions take the form of stationary and relative equilibria (traveling or rotating waves) [10]. Such solutions are called exact coherent states (ECSs) and express the minimal physical mechanisms behind turbulence sustainment [11]. Subcritical transition of canonical shear flows such as those in channels or pipes have been repeatedly shown to rely on the existence of ECSs [12]. Turbulent attractors arise from a sequence of instabilities of primal ECSs that originally appeared in saddle-node bifurcations. A satisfactory explanation of turbulence transition and sustainment in linearly stable TCF in terms of ECSs is still missing, while no other approach has yielded convincing results so far.

Newton-Raphson-based iterative methods have been used here in the search of ECSs within the linearly stable domain of TCF. Two spectral schemes, independently developed in [13] and [14], have been exploited concurrently. Both schemes discretize the Navier-Stokes equations by means of Fourier expansions in the axial and azimuthal coordinates, whereas the radial direction is approximated on a Chebyshev grid. The number of radial, azimuthal, and axial modes used to resolve ECSs in the both axially and azimuthally periodic domain are denoted $N_{r}, N_{\theta}$, and $N_{z}$, respectively, and systematic convergence tests have been performed with both schemes to ensure a satisfactory degree of spectral decay. Hereinafter we use $d=r_{o}-r_{i}$ and $\nu / d$ as length and velocity units, respectively. The nondimensional fundamental azimuthal and axial wave numbers of the ECSs, denoted by $n$ and $k$, respectively, define the periodic computational domain $\left[r_{i}, r_{o}\right] \times[0,2 \pi / n] \times[0,2 \pi / k]$.

A first attempt to identify subcritical ECSs in linearly stable TCF was undertaken in [15]. By choosing seeds emerging from linear instability, a number of primary (spiral) and secondary (ribbon) branches were shown to be only mildly subcritical.

In contrast, inspired by [10], the present study uses curvature homotopy to continue solutions from plane Couette flow [Fig. 1(b), the flow between exactly countersliding parallel plates, i.e., $\eta=1]$. Noting that artificially generated disturbances evolve into hairpin-shaped vortices in Rayleigh-stable Taylor-Couette experiments [16], we deem convenient to start the homotopy from a recently found plane Couette ECS featuring hairpin vortices [17] within its flow structure. Following the strategy of [13], the solution branch can be tracked successfully as it deforms from the flat to the curved geometry. The homotopic continuation procedure followed reduces $\eta$ while preserving the exact counterrotation speed of the cylinders throughout. The choice $\eta=0.883$ as the target allows comparison with the classical experimental studies of [4] and falls within the wide gap class as described in [18], where $\eta=\eta_{\text {crit }} \equiv 0.9 \sim 0.95$ is identified as a critical radius ratio involving a qualitative change in transition. Details of the homotopy path followed are not included here, as $\left(R_{o}, n, k\right)$ change in turns in such a contorted way that makes interpretation of the evolution of the solution along the path too complicated to be of interest. A simpler way of computing the new solutions is nevertheless provided later.

The resultant ECSs are characterized by a frozen threedimensional structure that travels in the azimuthal direction with angular wave speed $c$. The $R_{i}$ continuation of solution branches corresponding to several triplets $\left(R_{o}, n, k\right)$ undergo saddle-node bifurcations at $R_{i}=R_{i}^{\mathrm{SN}}\left(R_{o}, n, k\right)$ as shown in Fig. 3. Inner cylinder torque $T$, normalized by the CCF torque at the same parameter values, is used to measure deviation from CCF. For each value of $R_{o}$, the wave numbers $(n, k)$ are chosen so that the saddle-node point $R_{i}^{\mathrm{SN}}$ takes its minimal value. The spectral resolution used in the continuation is within the range $\left(N_{r}, N_{\theta}, N_{z}\right) \in$ $[38,52] \times[18,24] \times[26,30]$ to ensure convergence. Solid lines are ECS families found by homotopy from plane Couette flow. For $R_{o}=-1200$ (red solid line), the branch bifurcates in a saddle-node bifurcation that reaches a 


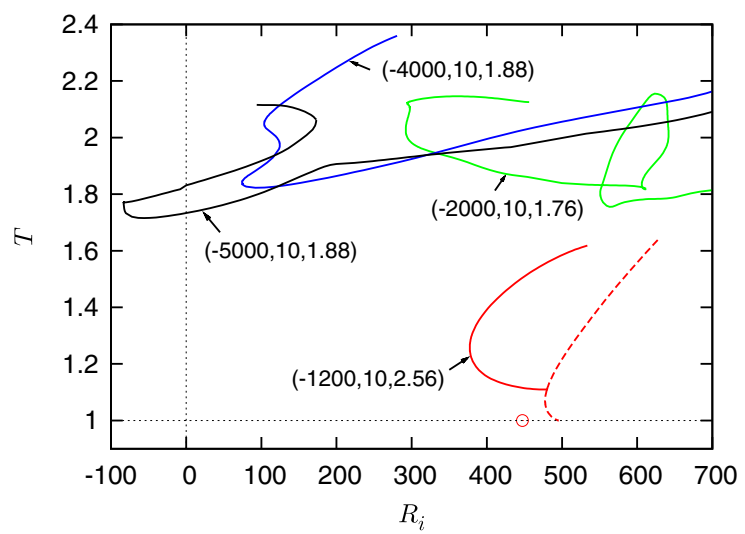

FIG. 3 (color online). Evolution of normalized (by CCF torque) inner cylinder torque $T$ along the $R_{i}$-continuation branches for fixed $\left(R_{0}, n, k\right)$ triplets. Solid lines represent ECS branches after curvature homotopy from a plane Couette solution. For each value of $R_{o}$, the wave numbers $n$ and $k$ minimize the saddle node $R_{i}^{S N}$. Also shown are the Taylor vortices branch $[(n, k)=(0,5.12)$, red dashed line $]$ and the linear critical point $\left[(n, k)=(5,5.125)\right.$, red open circle] for $R_{o}=-1200$. Note that all ECS branches are connected when the parameters are varied appropriately.

$\operatorname{minimum} R_{i}^{\mathrm{SN}} \approx 377.3$ for $(n, k)=(10,2.56)$. The primary linear instability of $\mathrm{CCF}$ for $R_{o}=-1200$ occurs at $R_{i} \simeq 447.4$ and corresponds to the bifurcation of a spiral mode with $(n, k)=(5,5.125)$ (red open circle). Although slightly subcritical, the spiral solution does not reach the low $R_{i}$ values of the homotopic ECSs [15] and therefore the ECSs qualify as remarkably subcritical. This subcriticality is enhanced as the outer cylinder is made to rotate faster (see $R_{o}=-2000,-4000$, and -5000 ; solid green, blue, and black lines, respectively). The minimal saddle-node point $R_{i}^{\mathrm{SN}}$ as a function of $R_{o}$ shows a clear trend in Fig. 2 (filled red circles connected by a red solid line): as the outer cylinder rotation rate is increased in modulus, the inner cylinder can be taken to lower counterrotation rates, eventually reaching the corotation regime as $\left|R_{o}\right|$ exceeds $\approx 4500$. The line defined by the saddle nodes runs parallel to and below the experimental thresholds found by [4]. This is compatible with the aforementioned scenario whereby a bifurcation cascade starting from an ECS originated at a saddle node gives rise to a turbulent set.

For $R_{o}=-1200$ the ECS solution branch connects to Taylor vortices [red dashed line, $(n, k)=(0,5.12)$, bifurcated from CCF at $\left.R_{i} \approx 480.0\right]$ in a symmetry-breaking bifurcation at $R_{i} \approx 495.6$. We have found no such connection for larger $\left|R_{o}\right|$. The resolution required to continue the solutions beyond the explored parameter ranges renders computations unaffordable. This connection suggests an alternative means of producing ECS solutions. Linear stability analysis of the Taylor vortices provides the eigenstate that must be added to the Taylor vortices in order to converge the ECS via Newton iteration. From this (a)

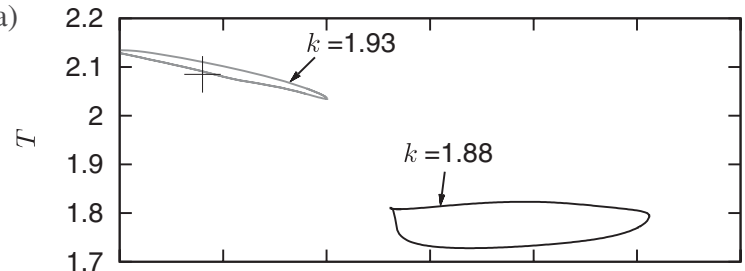

(b)

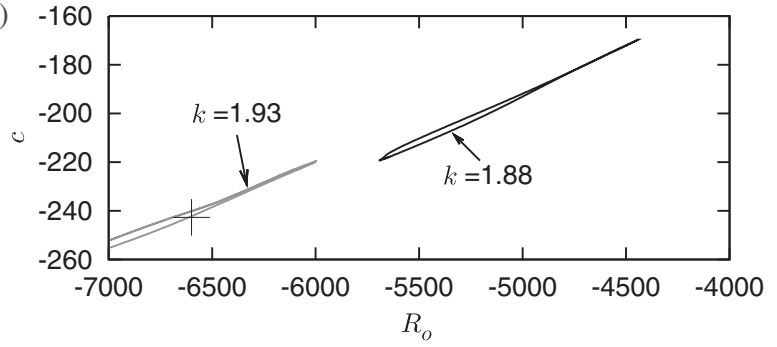

FIG. 4. Bifurcation diagram showing $R_{o}$-continuation ECS branches for $R_{i}=0$ and $n=10$. The wave number $k$ is chosen such that the rightmost saddle node $R_{o}^{S N}$ occurs closer to 0 . The plus sign represents a single solution computed at an increased resolution and used for flow field visualization. (a) Torque. (b) Angular wave speed.

first ECS branch at $\left(R_{i}, R_{o}, n, k\right)=(495.6,-1200$, 10,2.56), the rest of ECS branches in this Letter can simply be obtained by continuation.

Two distinct families of solution branches exist for $R_{i}=0$, as shown in Fig. 4. The pair of solutions at $R_{i}=0$ for the $R_{o}=-5000$ branch in Fig. 3 correspond to the upper and lower solution branches of the first family (black loop in Fig. 4). The second family (gray line in Fig. 4), which can only be computed for larger $\left|R_{o}\right|$, corresponds to a pair of extra crossings that are already

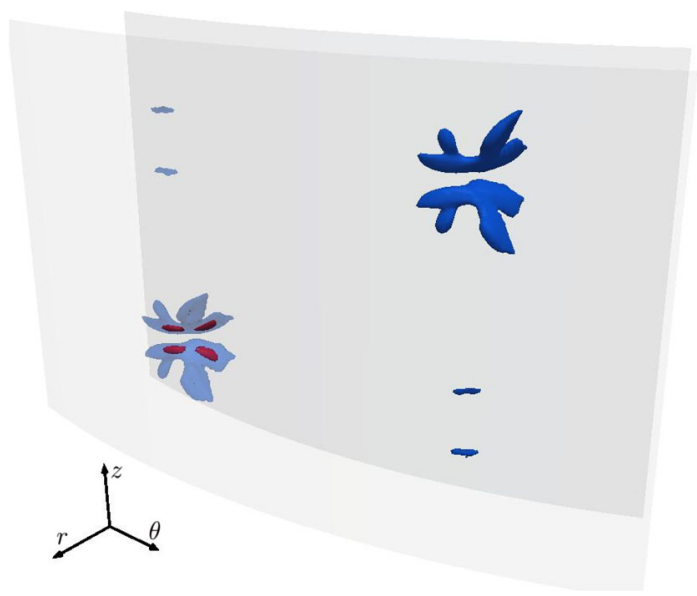

FIG. 5 (color online). Visualization of the localized vortex pattern of the ECS (plus sign of Fig. 4). The blue (dark gray) and red (light gray) isosurfaces implement the $\lambda_{2}$ criterion by showing $30 \%$ and $80 \%$ of the minimum negative value, respectively. The fundamental periodic domain $(n, k)=(10,1.93)$ is shown. The outer cylinder rotates leftward, while the inner cylinder is stationary. 


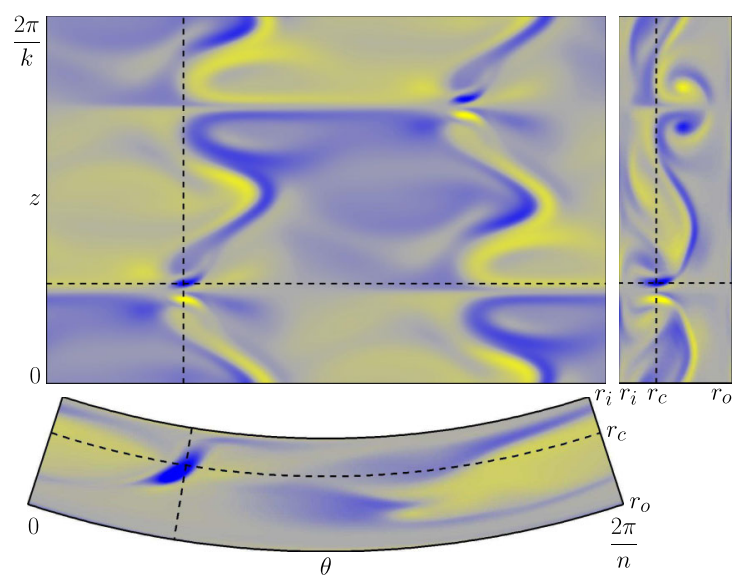

FIG. 6 (color online). Radial (top left), azimuthal (top right), and axial (bottom) cuts of the ECS (plus sign of Fig. 4) through the vortex core $(r, \theta, z)=\left(r_{c}, \theta_{c}, z_{c}\right)$ indicated by dashed lines. Areas of blue (dark) and yellow (light) represent positive and negative azimuthal vorticity.

hinted at by the $R_{o}=-5000$ branch in Fig. 3, where a further turning point issues a discontinued leftward-pointing upper branch. For $\left|R_{o}\right|$ in excess of 6000 and provided that $k$ is relaxed and allowed to increase up to 1.93 the solutions can be continued back to $R_{i}=0$.

The solutions present a strongly localized structure that calls for a very fine discretization. A single refinement with a high resolution of $\left(N_{r}, N_{\theta}, N_{z}\right)=(56,50,60)$ (the plus sign in Fig. 4; torque $T=2.076$, angular wave speed $c=242.7$ ) results in a very accurate spectral convergence, thus confirming that the solutions are physical and that their main features are already captured with the lower resolutions. The localized vortical structure of the solution is shown in Fig. 5, where the $\lambda_{2}$ criterion [19] has been used to identify vortex cores. While only one axial or azimuthal wavelength is shown, periodicity results in a staggered pattern of vortex pairs when considering the full annulus and a long-aspect ratio apparatus.

Figure 6 shows radial, axial, and azimuthal sections through the leftmost vortex core in Fig. 5. The radial cut of azimuthal vorticity contours reveals the staggered arrangement of vortex pairs, clearly identifiable as a shift-rotate symmetry $[u, v, w](r, \theta, z)=[u, v, w](r, \theta+\pi / n, z+\pi / k)$, where $(u, v, w)=u \hat{\mathbf{r}}+v \hat{\boldsymbol{\theta}}+w \hat{\mathbf{z}}$ are the radial, azimuthal, and axial velocity components. The solution also exhibits a mirror symmetry $[u, v, w]\left(r, \theta, z+z_{0}\right)=[u, v,-w]$ $\left(r, \theta,-z+z_{0}\right)$, with respect to two $z$ planes at $z_{0}=\pi / 2 k$ and $3 \pi / 2 k$ traversing the space in between each localized vortex pair. Note that $\lambda_{2}$ and azimuthal vorticity are even and odd upon $z$ reflection, respectively. The localized vortex is congruent with experimentally generated vortices in [16], where agreement with the theoretical predictions of [20] is reported. The tilt angle of the localized vortex of Fig. 6 ( $z$ cut $)$ is in excellent agreement with the theoretical value $\phi \approx 40^{\circ}$ predicted by considering the localized

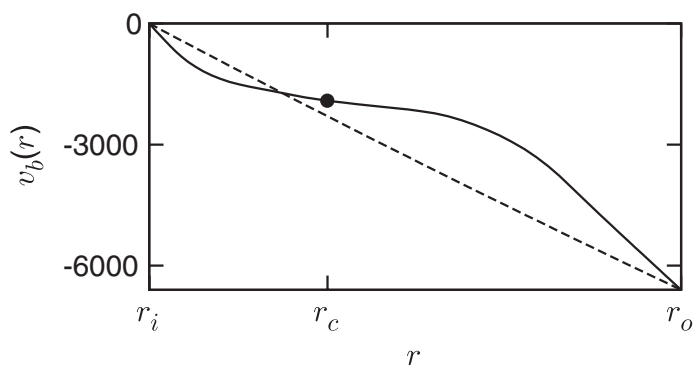

FIG. 7. Radial profiles of background azimuthal velocity of the ECS (solid line, the plus sign of Fig. 4, resulting from axialazimuthal averaging across the domain). The filled circle indicates the critical radius $r=r_{c}$ at which $v_{b}$ equals the rotation speed $r_{c} c$ of the ECS. The laminar CCF profile is shown as a dashed line for comparison.

disturbance as developing on a background flow described by the $\theta-z$ averaged velocity field $v_{b}(r)$ shown in Fig. 7. The critical radius $r=r_{c} \approx 7.882$ crosses right through the vortex core, as is evident from Fig. 6, which means that the vortex has developed within the thin layer around $r=r_{c}$ and is advected with the average flow $v_{b}\left(r_{c}\right)$.

The mirror symmetry of the solutions presented in this Letter is likely to be essential to the origin and sustainment of nonlaminar states in highly subcritical TCF, given that the non-mirror-symmetric plane Couette solutions of [10] yield but mild subcriticality when continued to TCF. Furthermore, the staggered pattern of localized vortex pairs is compatible with the observed fact that oblique-banded structures typical of narrow-gap stationary inner cylinder TCF collapse into intermittency when $\eta$ falls below $\eta_{\text {crit }}$.

In summary, our investigation uncovers the existence of nonlinear solutions that have all the features necessary for the sustainment of turbulence in highly subcritical TCF. The solutions, which may comprise the backbone of the chaotic set that is responsible for turbulence, are driven by the effect of shear as settled by the fact that they subsist even in the Rayleigh stable regime. At the other end, these solutions connect to Taylor vortices of centrifugal origin. Analyzing how our highly subcritical solutions evolve as centrifugal forces take over might be of relevance in elucidating the essential mechanisms behind centrifugal and shear instabilities of rotating flows.

This work was partially supported by the JSPS Institutional Program for Young Researcher Overseas Visits. The research of A.M. and F. M. was supported by Grants No. FIS2009-08821 and No. 2009SGR1045 from the Spanish and Catalan governments, respectively.

\footnotetext{
*k.deguchi418@gmail.com

†fernando.mellibovsky@upc.edu

[1] R. Tagg, Nonlinear Sci. Today 4, 1 (1994).
} 
[2] L. Rayleigh, Proc. R. Soc. A 93, 148 (1917).

[3] E. L. Koschmieder, Bénard Cells and Taylor Vortices, Cambridge Monographs on Mechanics and Applied Mathematics (Cambridge University Press, Cambridge, England, 1989).

[4] C. D. Andereck, S. S. Liu, and H. L. Swinney, J. Fluid Mech. 164, 155 (1986); D. Coles, J. Fluid Mech. 21, 385 (1965).

[5] H. Ji, M. Burin, E. Schartman, and J. Goodman, Nature (London) 444, 343 (2006).

[6] D. Borrero-Echeverry, M. F. Schatz, and R. Tagg, Phys. Rev. E 81, 025301(R) (2010).

[7] M. Avila, Phys. Rev. Lett. 108, 124501 (2012); M. Heise, Ch. Hoffmann, Ch. Will, S. Altmeyer, J. Abshagen, and G. Pfister, J. Fluid Mech. 716, R4 (2013).

[8] A. Meseguer, Phys. Fluids 14, 1655 (2002); A. Meseguer, F. Mellibovsky, M. Avila, and F. Marques, Phys. Rev. E 80, 046315 (2009).

[9] F. Mellibovsky and B. Eckhardt, J. Fluid Mech. 709, 149 (2012); T. Kreilos and B. Eckhardt, Chaos 22, 047505 (2012).

[10] M. Nagata, J. Fluid Mech. 217, 519 (1990); M. Nagata, Phys. Rev. E 55, 2023 (1997).
[11] F. Waleffe, Phys. Fluids 9, 883 (1997).

[12] T. Itano, and S. Toh, J. Phys. Soc. Jpn. 70, 703 (2001); B. Hof, C. W. H. van Doorne, J. Westerweel, F. T. M. Nieuwstadt, H. Faisst, B. Eckhardt, H. Wedin, R. R. Kerswell, and F. Waleffe, Science 305, 1594 (2004); J. F. Gibson, J. Halcrow, and P. Cvitanovic, J. Fluid Mech. 611, 107 (2008).

[13] K. Deguchi, and M. Nagata, J. Fluid Mech. 678, 156 (2011).

[14] A. Meseguer, M. Avila, F. Mellibovsky, and F. Marques, Eur. Phys. J. Spec. Top. 146, 249 (2007).

[15] A. Meseguer, F. Mellibovsky, M. Avila, and F. Marques, Phys. Rev. E 79, 036309 (2009); K. Deguchi and S. Altmeyer, Phys. Rev. E 87, 043017 (2013).

[16] E. Malkiel, V. Levinski, and J. Cohen, J. Fluid Mech. 379, 351 (1999).

[17] T. Itano and S. C. Generalis, Phys. Rev. Lett. 102, 114501 (2009); J. F. Gibson, J. Halcrow, and P. Cvitanovic, J. Fluid Mech. 638, 243 (2009).

[18] D. Richard and J.-P. Zahn, Astron. Astrophys. 347, 734 (1999); M. J. Burin and C. J. Czarnocki, J. Fluid Mech. 709, 106 (2012).

[19] J. Jeong and F. Hussain, J. Fluid Mech. 285, 69 (1995).

[20] V. Levinski and J. Cohen, J. Fluid Mech. 289, 159 (1995). 